\title{
Giant arachnoid granulation in a patient with benign intracranial hypertension (2009: 1a)
}

(C) European Society of Radiology 2008

\section{Case report}

A 48-year-old moderately obese man with chronic headache and vertigo was referred to magnetic resonance (MR) examination. Although he had a past history of visual disturbance, his recent visual examination was normal. His neurologic examination was not notable. A lumbar puncture showed a mildly increased opening pressure $(23 \mathrm{cmHg})$, and the laboratory findings were normal. MR images showed no parenchyma abnormalities whereas a nodular filling defect in his superior sagittal sinus (Fig. 1).

\section{What is the diagnosis?}

Readers are invited to supply one possible diagnosis via electronic means to: robert.hermans@uzleuven.be.
The subject of the email should include 'Interpretation Corner' and the number 2009: 1 a.

You should include your name, title, address, fax, and phone number.

Deadline: one clear calendar month from distribution date.

Three months after the initial publication of the case history, the authors will publish the final diagnosis and a brief summary. The summary will describe exactly how the case was investigated at the host institution, how the diagnosis was established, and the teaching points of the case in question.

The names of the first 25 radiologists submitting the correct diagnosis will be published. (Only one from any individual center and none from the host institution!)
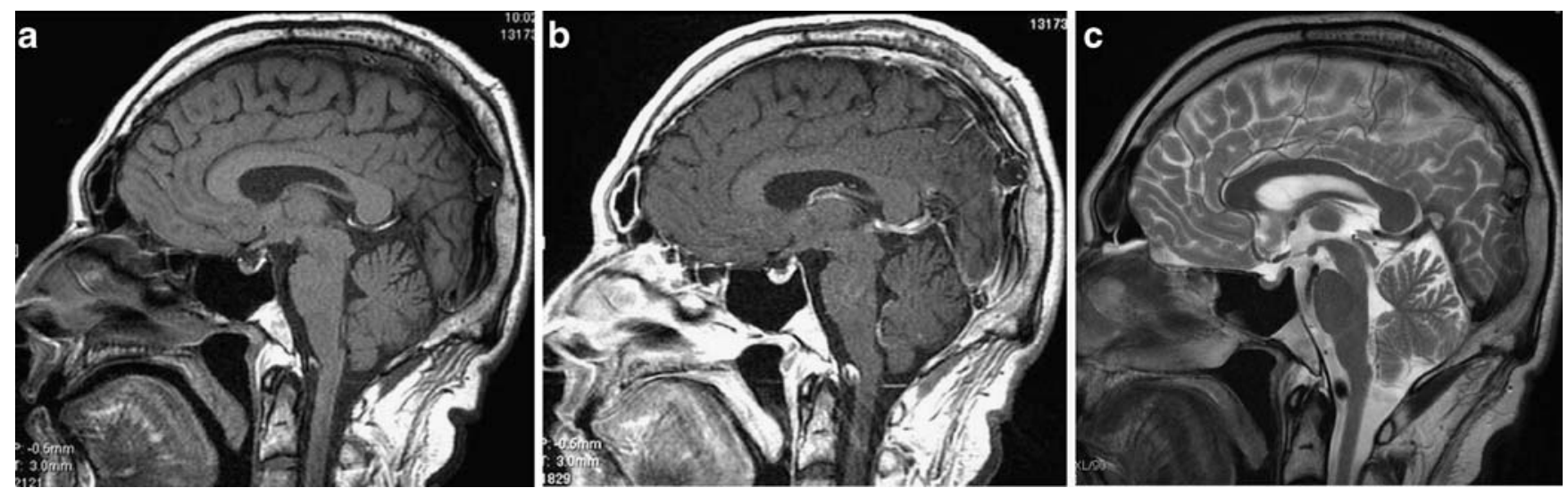

Fig. 1 Sagittal MR images of the patient with benign intracranial hypertension. Plain (a) and gadolinium-enhanced T1-weighted (b) images, T2-weighted image (c) 